\title{
Determination of follistatin-like protein 1 expression in colorectal cancer and its association with clinical outcomes
}

\author{
Yujie Zhao ${ }^{1 \#}$, Qingjian Ou ${ }^{1 \#}$, Yuxiang Deng ${ }^{1 \#}$, Jianhong Peng ${ }^{1}$, Cong Li $^{1}$, Jibin Li $^{2}$, Qian Zhao ${ }^{3}$, \\ Miaozhen Qiu ${ }^{4}$, Desen Wan ${ }^{1}$, Yujing Fang ${ }^{1}$, Zhizhong Pan ${ }^{1}$ \\ ${ }^{1}$ Department of Colorectal Surgery, ${ }^{2}$ Department of Clinical Research, State Key Laboratory of Oncology in South China, Collaborative Innovation \\ Center for Cancer Medicine, Sun Yat-sen University Cancer Center, Guangzhou 510060, China; ${ }^{3}$ Department of Statistics, School of Public Health, \\ Guangzhou Medical University, Guangzhou 511436, China; ${ }^{4}$ Department of Medical Oncology, State Key Laboratory of Oncology in South China, \\ Sun Yat-sen University Cancer Center, Guangzhou 510060, China. \\ Contributions: (I) Conception and design: Y Zhao, Q Ou; (II) Administrative support: Y Fang, Z Pan; (III) Provision of study materials or patients: \\ Y Deng, Q Zhao; (IV) Collection and assembly of data: J Li, M Qiu, D Wan; (V) Data analysis and interpretation: J Peng, C Li; (VI) Manuscript \\ writing: All authors; (VII) Final approval of manuscript: All authors. \\ \#These authors contributed equally to this work. \\ Correspondence to: Yujing Fang; Zhizhong Pan. Department of Colorectal Surgery, State Key Laboratory of Oncology in South China, Collaborative \\ Innovation Center for Cancer Medicine, Sun Yat-sen University Cancer Center, 651 Dongfeng East Road, Guangzhou 510060, China. \\ Email: fangyj@sysucc.org.cn; panzhzh@sysucc.org.cn.
}

Background: Follistatin-like protein 1 (FSTL1) has been demonstrated to play a controversial role in cancer. In this study, we aimed to investigate the expression of FSTL1 and its characteristics in patients with colorectal cancer (CRC).

Methods: Gene expression microarray assays in $30 \mathrm{CRC}$ patients and a real-time quantitative polymerase chain reaction (RT-qPCR) of 22 patients were performed to compare the mRNA level of FSTL1 in tumor lesions and paired normal tissues. Also, 332 consecutive patients with pathologically confirmed CRC were selected to detect FSTL1 expression by using immunohistochemistry (IHC). Enzyme-linked immunosorbent assay (ELISA) was also applied to determine the serum level of FSTL1 in 60 CRC patients, as well as 34 healthy donors.

Results: Gene expression microarray assays and RT-qPCR in CRC tissues, as well as ELISA in the serum all, revealed that the expression level of FSTL1 was higher in cancer tissue of CRC patients compared with paired normal tissue or healthy donors. The IHC results suggested that FSTL1 was also higher in tumor tissues than in its normal counterparts, however interestingly, a narrow scan focusing on the stromal region indicated that FSTL1 was significantly higher in normal tissues than in cancerous tissues. Besides, higher FSTL1 expression in cancer tissue, as well as lower FSTL1 expression in cancer stroma, both correlated with a worse prognosis, and the latter was an independent prognostic factor.

Conclusions: Our results provide novel insight into the role of FSTL1 in CRC, and it might be an essential factor in CRC development.

Keywords: Colorectal cancer (CRC); follistatin-like protein 1 (FSTL1); stroma; prognosis

Submitted Aug 08, 2019. Accepted for publication Aug 19, 2019.

doi: $10.21037 /$ atm.2019.09.20

View this article at: http://dx.doi.org/10.21037/atm.2019.09.20 


\section{Introduction}

Colorectal cancer (CRC) is the fifth leading cause of cancerrelated death in China, with a total of 191,000 deaths in 2015 (1). Although risk factors such as T4 tumors, the elevation of preoperative carcinoembryonic antigen (CEA) levels, and the presence of lymphovascular or perineural invasion and mesenteric tumor nodules have been demonstrated to be factors for a poor clinical outcome, this information is still insufficient for precise clinical treatment $(2,3)$. Therefore, the exploration of new biomarkers is essential to distinguish subgroups with a high risk of recurrence and to personalize therapy regimens.

Follistatin-like protein 1 (FSTL1), which is also called TSC-36, is a secreted glycoprotein encoded on chromosome 3 in humans that is widely expressed in cells of nonhematopoietic lineage, particularly in cells of mesenchymal lineage (4). The function of FSTL1 has been partially elucidated in several developed tissue types. It has been reported that FSTL1 participates in the regulation of autoimmune diseases such as rheumatoid arthritis (5). In joint tissue, FSTL1 decreases the production of matrix metalloproteinases and prostaglandins from synovial fibroblasts, which modifies joint inflammation and destruction in arthritic disease (6,7). In blood cells, graft-infiltrating CD8 $+\mathrm{T}$ cells produce FSTL1, which contributes to allograft tolerance (8). In cardiovascular tissue, FSTL1 inhibits smooth muscle cell proliferation and migration and protects cardiac myocytes from hypoxia/ reoxygenation-induced apoptosis $(9,10)$. However, the role of FSTL1 in cancer is controversial. FSTL1 is frequently downregulated and plays a tumor suppressor role in metastatic clear cell renal cell carcinoma (ccRCC) (11). In prostate cancer, the androgen-dependent upregulation of FSTL1 promotes the growth of cancer cells (12). When cancer metastasizes from the primary site to the bone, FSTL1 can facilitate the invasion by cancer cells and expand bone marrow-derived pluripotent mesenchymal stemlike cells (13). In ovarian and endometrial cancers, FSTL1 functions as a tumor suppressor by inducing apoptosis (14), while in lung cancer cells, its inhibition can promote mitotic cell death via inactivated Erk1/2 (15).

Although FSTL1 is overexpressed in the plasma and cancerous tissues of CRC patients (16), FSTL1 has not been implicated in prognosis. Previous studies have found that FSTL1, which is expressed in cancer stroma, can attenuate CRC cell proliferation (17). However, the expression of FSTL1 protein in CRC has not yet been well elucidated. In the present study, we aimed to explore the level of FSTL1 expression in the tissue and peripheral plasma of CRC patients and to validate the prognostic functions of FSTL1 in CRC patients.

\section{Methods}

\section{Clinical samples}

All clinical samples were collected from the Sun Yat-sen University Cancer Center (Guangzhou, China) (Figure 1). For the transcriptome array, 30 pairs of fresh-frozen CRC and adjacent nontumor tissue specimens were randomly selected. Of the 30 patients, 18 patients had stage I to III CRC (6 patients per stage), and 12 patients had stage IV CRC. Twenty-two primary human CRC and adjacent nontumor colorectal tissue samples were collected and used for quantitative polymerase chain reactions (RT-qPCR) in FSTL1 expression studies. In all, 310 CRC and 247 nontumor colorectal formalin-fixed, paraffin-embedded tissue specimens were obtained from a total of 332 patients who underwent colectomy at Sun Yat-sen University Cancer Center and were used for tissue microarray (TMA) construction and immunohistochemistry (IHC) analysis. All included tumor specimens were histologically confirmed to be colorectal adenocarcinoma. Patients were excluded from the analysis if they died within one month of surgery, had incomplete pathological data, or were lost to follow-up within three months. Patient demographics and clinicopathological characteristics were retrieved from the medical records, and follow-up data were collected from the tracking system. Serum samples from a total of 60 CRC patients and 34 normal healthy individuals were collected and used for enzyme-linked immunosorbent assay (ELISA) analysis of secretory FSTL1 expression.

\section{Transcriptome array}

RNA isolation from the clinical samples, microarray analysis, data processing, statistical analysis, and gene ontology analysis was performed by Shanghai Biotechnology Corporation (Shanghai, China). The microarray experiments were performed using an Affymetrix GeneChip Human Transcriptome Array 2.0 (HTA2.0).

\section{$R T-q P C R$}

Total cellular RNA was extracted from frozen samples using TRIzol reagent (Invitrogen, CA, USA). Reverse transcription (RT) was performed using a Revert Aid First 


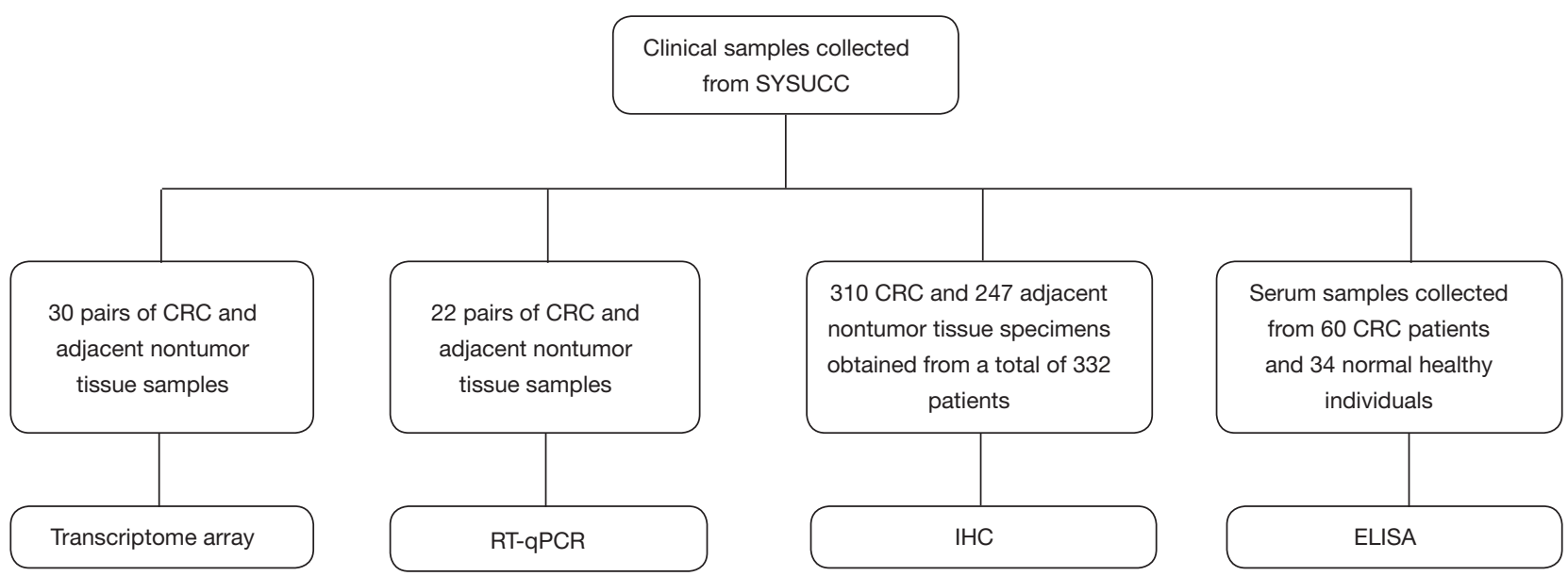

Figure 1 Flow diagram summarizing the patients involved in our study. Clinical samples of tissues and serum collected from Sun Yatsen University Cancer Center (SYSUCC) were classified into 4 groups for determination of FSTL1 expression via transcriptome array, RT-qPCR, IHC and ELISA. The included CRC patients had received no previous local or systemic treatment prior to surgery. FSTL1, follistatin-like protein 1; RT-qPCR, real-time quantitative polymerase chain reaction; IHC, immunohistochemistry; ELISA, enzyme-linked immunosorbent assay; CRC, colorectal cancer.

Strand cDNA Synthesis Kit (Thermo Scientific, Waltham, MA, USA) according to the manufacturer's instructions. RT-qPCR amplification was performed with DreamTaq DNA Polymerase (Thermo Scientific, Waltham, MA, USA) using a ROCHE 480 system. The primer sequences were FSTL1: F (5'-CCCAGTTGTTTGCTATCAGTCC-3') and R (5'-TGTAGTTGCTGCCTTTAGAGAAC-3') and $\beta$-actin: F (5'-GCACTCTTCCAGCCTTCC-3') and R (5'-TGTCCACGTCACACTTCATG-3'). Relative expression differences were calculated using the $2^{-\Delta \Delta C t}$ methods.

\section{ELISA}

Serum FSTL1 in both CRC patients and healthy donors was quantified by ELISA using a human FSTL1 ELISA Kit (BOSTER, California, USA), as described by the manufacturer. The results are expressed in $\mathrm{ng} / \mathrm{mL}$, and the optical density of the samples was compared with the standard curves. The secretory FSTL1 levels in the serum were compared between CRC patients and healthy donors.

\section{TMAs and IHC}

The TMAs were constructed using a personal tissue array (Beecher Instruments, Sun Prairie, WI, USA). Tumor tissues and normal counterparts were obtained from each patient, with 3 to 4 cores per tissue. The diameter of each core was $1 \mathrm{~mm}$. The organized TMA blocks were sectioned into 4- $\mu \mathrm{m}$-thick slices that were mounted onto glass slides. After deparaffinization, the slides were treated with $0.3 \%$ hydrogen peroxide and incubated with a primary FSTL1 antibody (1:500 dilution; Abgent no. AP10534b, CA, USA) in a humidified chamber at $4{ }^{\circ} \mathrm{C}$ overnight. Subsequently, the slides were washed with $1 \times$ phosphate-buffered saline (PBS) and treated with a biotinylated anti-rabbit secondary antibody (Zhongshan Golden Bridge Biotechnology, Beijing, China) at $37.5^{\circ} \mathrm{C}$ for $30 \mathrm{~min}$. IHC staining was completed by incubation with 3,3'-diaminobenzidine tetrahydrochloride (DAB; Dako, Glostrup, Denmark) to stain the slides.

\section{IHC score}

The IHC score was determined by a semiquantitative method based on the percentage and intensity of positively stained cells. The percentage of positively stained cells was scored as follows: 0 , less than $5 \%$ positively stained cells; 1, 5-24\%; 2, 25-49\%; 3, 50-74\%; and 4, 75-100\%. The intensity was scored according to the following criteria: 0 , negative staining; 1 , weak staining; 2 , moderate staining; and 3, strong staining. The final IHC score was generated by multiplying the percentage score with the staining intensity score. Each slide was immunohistochemically 


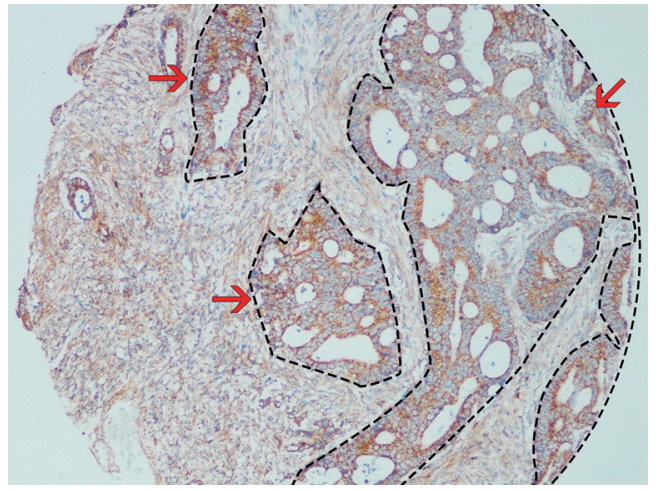

Figure 2 The example of image analysis of IHC staining in constructed TMAs. The area inside the dotted line belong to the colorectal epithelium (indicated by the red arrow). Each slide experienced evaluations twice, one for epithelial as well as stroma area (the full scan, i.e. within and outside the dashed line), and the other for only the stroma area (the narrow scan, i.e., outside the dotted line). IHC, immunohistochemistry; TMA, tissue microarray.

analyzed from two aspects, one for staining the entire slide includes both epithelial and stroma staining (full scan) and the other for only the stroma fraction (narrow scan). The example of image analysis was shown in Figure 2. Evaluation of FSTL1 expression was performed by two pathologists independently who had no prior knowledge of the patient data. The receiver operating curve (ROC) analysis was applied to calculate the area under the ROC curve (AUC) to determine the optimal cutoff values for the FSTL1 expression level. High FSTL1 expression grade was defined when the IHC score was greater than the optimal cutoff value. Both the expression levels in the full scan of tumor tissues and normal counterparts and a narrow scan focusing on the stromal region were considered.

\section{Statistical analysis}

Statistical analyses were performed using SPSS 20.0 (IBM, Chicago, USA) and GraphPad Prism 7 (GraphPad Software, Inc, CA, USA) software. We compared continuous variables that were normally distributed using Student's $t$-test and categorical variables using a chi-square $\left(\chi^{2}\right)$ test, Fisher's test, or nonparametric Mann-Whitney U test. The KaplanMeier method was used to estimate the survival rates of the different groups, and differences in survival were compared using a log-rank test. In regards to the statistically significant variables, where $\mathrm{P}<0.05$ in univariate $\mathrm{Cox}$ models, were further assessed with multivariate Cox models using a stepwise forward method. Hazard ratios (HRs) and $95 \%$ confidence intervals $(\mathrm{CI})$ were calculated. $\mathrm{P}<0.05$ was considered statistically significant. The authenticity of this article has been validated by uploading the key raw data onto the Research Data Deposit public platform (http:// www.researchdata.org.cn), with the approval number RDDB2018000386.

\section{Results}

\section{Transcriptome array and RT-qPCR analysis of FSTL1 in CRC and tumor-adjacent normal tissues}

Transcriptome arrays with 30 pairs of matched tumors and adjacent nontumor tissues from patients with CRC were analyzed. Increased expression of FSTL1 was investigated in 30 CRC samples and was compared with the expression in corresponding nontumor tissues (Figure $3 A$; mean $\pm \mathrm{SD}$ : $9.12 \pm 1.32$ vs. $7.94 \pm 0.91, \mathrm{P}<0.001)$. To determine whether upregulation of FSTL1 is a common event, RT-qPCR was performed on an additional 22 pairs of primary human CRC and adjacent nontumor colorectal tissue samples to validate our findings, and the results were concordant with the previous results (Figure 3B; mean $\pm \mathrm{SD}$ : $12.37 \pm 1.95$ vs. $10.46 \pm 1.58, \mathrm{P}=0.002)$.

\section{Serum FSTL1 expression in CRC patients and healthy donors}

It has been demonstrated that FSTL1 is a secretory glycoprotein since it can be detected in the serum and synovial fluid of patients with rheumatoid arthritis (18). Serum samples from 34 healthy individuals and 60 CRC patients were collected for ELISA analysis to investigate whether serum FSTL1 levels were different between CRC patients and healthy individuals. A marked difference between the two groups was observed in the FSTL1 serum levels: FSTL1 expression was significantly higher in CRC patients than in healthy controls (Figure $3 C$; mean $\pm \mathrm{SD}$ : $1.91 \pm 1.40$ vs. $1.10 \pm 1.25, \mathrm{P}<0.001)$.

\section{Expression of FSTL1 in CRC and tumor-adjacent normal tissues revealed by an IHC analysis of TMAs}

The levels of FSTL1 protein were examined via IHC using TMAs from a total of 332 consecutive patients with pathologically confirmed stage II-IV CRC; the TMAs 
A
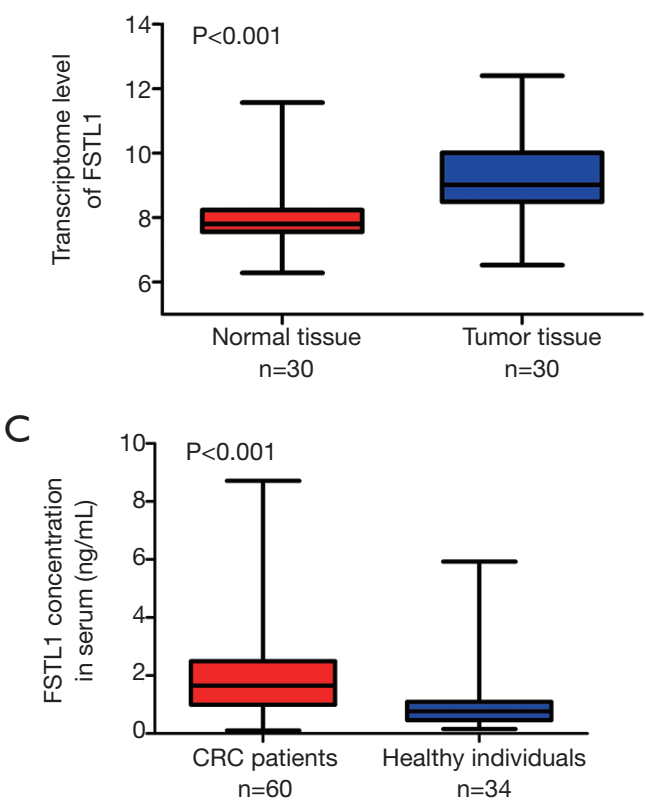

B

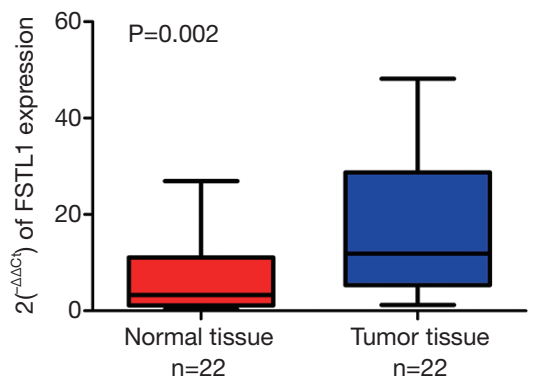

Figure 3 FSTL1 is frequently overexpressed in CRC samples both for tumor tissues and serum. (A) Expression of FSTL1 in 30 pairs of CRC and corresponding nontumor tissues [18 patients with stage I to III CRC (6 patients per stage), and 12 patients with stage IV CRC (6 patients with liver-only metastasis and 6 patients with metastasis in organs other than the liver)] examined by transcriptome array (mean \pm SD: $9.12 \pm 1.32$ vs. 7.94 $\pm 0.91, \mathrm{P}<0.001$ ). (B) Expression of FSTL1 in 22 pairs of tumors and nontumor counterparts examined via RT-qPCR (mean \pm SD: $12.37 \pm 1.95$ vs. 10.46 $\pm 1.58, \mathrm{P}=0.002$ ). (C) Box plot showing the ELISA results of the expression of FSTL1 in sera collected from 34 healthy normal individuals and $60 \mathrm{CRC}$ patients (mean $\pm \mathrm{SD}: 1.91 \pm 1.40$ vs. $1.10 \pm 1.25, \mathrm{P}<0.001$ ). FSTL1, follistatin-like protein 1; RTqPCR, real-time quantitative polymerase chain reaction; IHC, immunohistochemistry; ELISA, enzyme-linked immunosorbent assay; CRC, colorectal cancer.

contained 247 nontumor tissues and 310 CRC tissues. FSTL1 was shown to be present in both epithelial cells and tumor stroma (Figure 4). As in the full scans, we found that FSTL1 was highly expressed in the majority of the CRC samples compared with their normal counterparts (Figure 5 A; $7.39 \pm 3.18$ vs. $3.09 \pm 2.38, \mathrm{P}<0.001$ ), but a narrow scan that focused on the stromal region indicated that FSTL1 expression was significantly higher in the normal tissues than in the cancerous tissues (Figure $5 B ; 6.34 \pm 2.60$ vs. $3.71 \pm 2.18, \mathrm{P}<0.001)$.

\section{Association of FSTL1 expression in different regions of tumor tissue with clinicopathological features}

In all, 310 patients whose tumor tissues were analyzed for FSTL1 expression were included in the present study. As shown in Table 1, the median age of all patients was 59 years (range, 23-91 years); 61.9\% (192/310) were males, and $38.1 \%$ (118/310) were females. Of the 310 patients, 203 patients were diagnosed with colon cancer, and 107 were diagnosed with rectal cancer. Concerning the TNM stage, $101(32.6 \%)$ patients were diagnosed with stage II CRC and 209 (67.4\%) with stage III/IV CRC. For the histological grade, $83.9 \%(260 / 310)$ patients had well/moderately differentiated adenocarcinoma, and $16.1 \%(50 / 310)$ had poorly differentiated CRC.

In different regions of tumor tissue, we analyzed the association between FSTL1 expression and the clinicopathological features of CRC patients. The optimal IHC score cutoff values for FSTL1 expression in tumor tissues assessed via a full scan and a narrow scan of the tumor stroma were 6.5 and 3.17 , respectively, as determined by ROC curve analysis (Figure 6). The patients were further divided into two groups, as follows: 131 (42.3\%) patients were in the low FSTL1 expression group after full tumor tissue scan (IHC score $\leq 6.5$ ), and 179 (57.7\%) patients were in the high expression group (IHC score $>6.5$ ). For FSTL1 expression in the tumor stroma, 163 

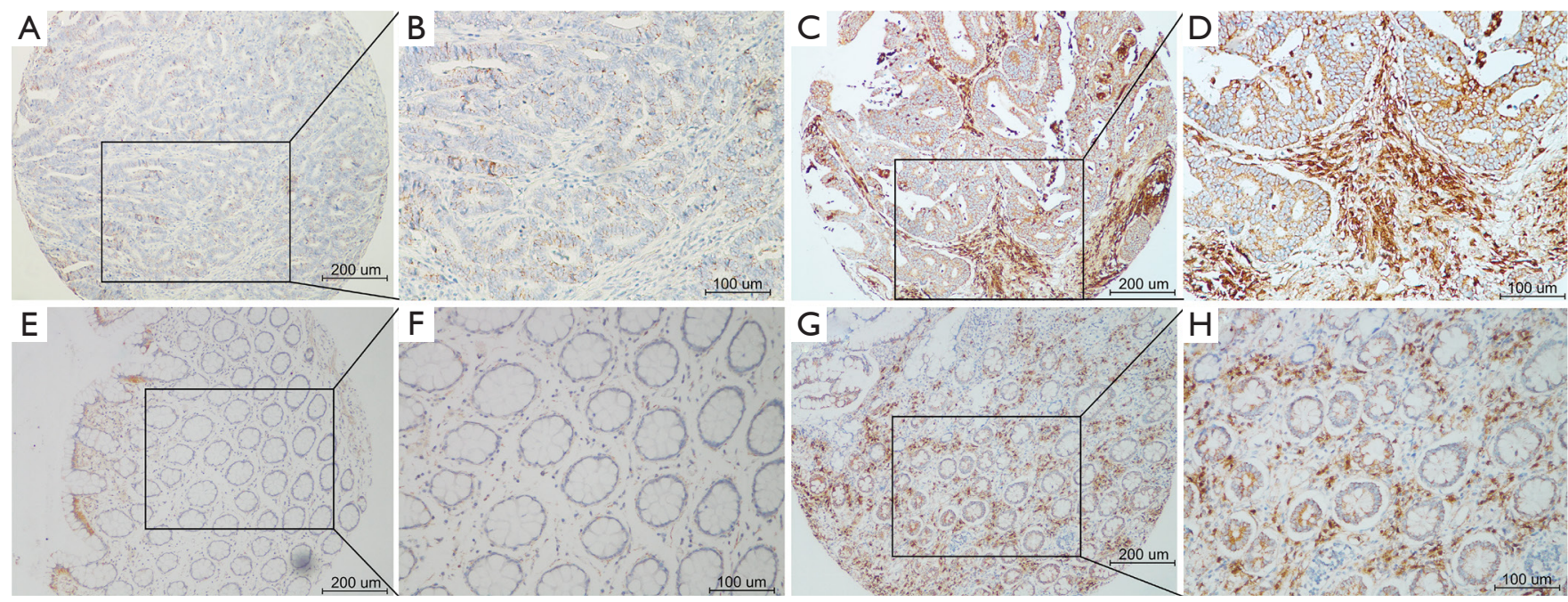

Figure 4 Representative IHC for FSTL1 in full scans and narrow scans of the stroma in tumor tissues and tumor-adjacent normal tissues. Scale bar, 200 and $100 \mu \mathrm{m}$. (A,B) CRC tissue with weak FSTL1 expression in both the full scan and the stromal region; (C,D) CRC tissue with strong FSTL1 expression (especially in the stroma); (E,F) weak FSTL1 expression in tumor-adjacent normal tissue in both the full scan and in the stroma; $(\mathrm{G}, \mathrm{H})$ strong FSTL1 expression in tumor-adjacent normal tissue (especially in the stroma). FSTL1, follistatin-like protein 1; IHC, immunohistochemistry; CRC, colorectal cancer.
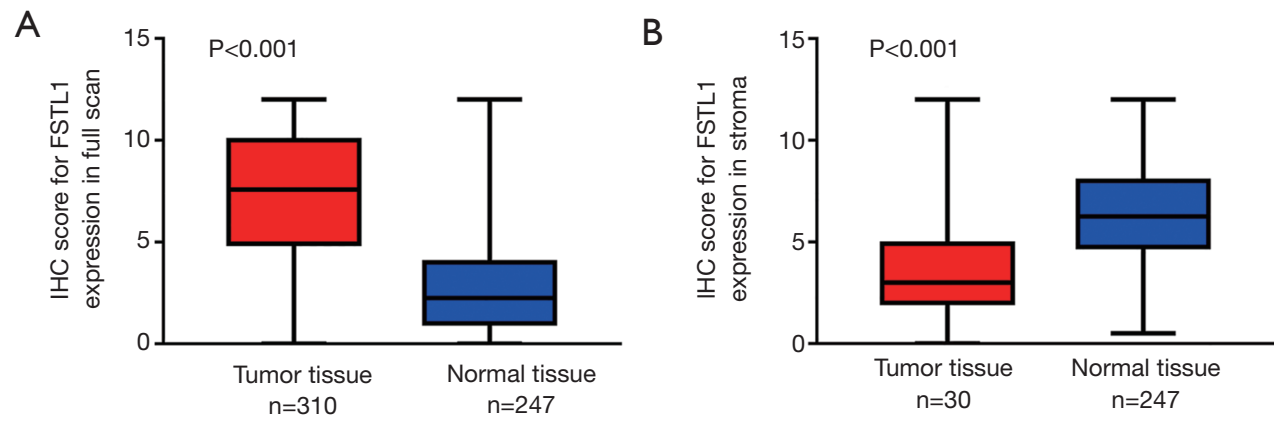

Figure 5 Bar graph summary of the distribution of FSTL1 expression levels in nontumor $(\mathrm{n}=247) v s$. CRC (n=310) tissues examined via IHC of TMA ( $\mathrm{n}=332$ total cases). (A) Comparison of FSTL1 expression levels between fully scanned CRC tissues and tumor-adjacent normal tissues (mean \pm SD: $7.39 \pm 3.18$ vs. 3.09 $\pm 2.38, \mathrm{P}<0.001$ ); (B) comparison of FSTL1 expression levels shown on a narrow scan of the stromal region between tumor and tumor-adjacent normal tissues (mean \pm SD: $3.71 \pm 2.18 v s .6 .34 \pm 2.60$, P<0.001). FSTL1, follistatin-like protein 1; IHC, immunohistochemistry; CRC, colorectal cancer; TMA, tissue microarray.

$(52.6 \%)$ patients were in the low expression group (IHC score $\leq 3.17)$, while 147 (47.4\%) were in the high expression group (IHC score $>3.17$ ). The associations between FSTL1 expression in different regions and the clinicopathological parameters, including gender, age, tumor location, tumor size, histological type, clinical stage, and preoperative serum CEA and CA19-9 levels, were assessed (Table 2). Tumor tissue FSTL1 expression in the full scan was found to be associated with primary tumor site, TNM stage, and
CA19-9 level $(\mathrm{P}<0.05)$. Additionally, FSTL1 expression in the tumor stroma was associated only with the primary tumor location $(\mathrm{P}<0.001)$, whereas associations with other clinicopathological features were not observed $(\mathrm{P}>0.05)$.

\section{Association of FSTL1 expression in different regions of tumor tissue with long-term survival}

During a median follow-up of 50.35 months (range, 
Table 1 Clinicopathologic features of patients involved in this study

\begin{tabular}{|c|c|}
\hline Characteristics & Total $(n=310), n(\%)$ \\
\hline \multicolumn{2}{|l|}{ Age (years) } \\
\hline$\leq 59$ & $161(51.9)$ \\
\hline$>59$ & $149(48.1)$ \\
\hline \multicolumn{2}{|l|}{ Sex } \\
\hline Female & $118(38.1)$ \\
\hline Male & $192(61.9)$ \\
\hline \multicolumn{2}{|l|}{ Primary tumor site } \\
\hline Colon & $203(65.5)$ \\
\hline Rectum & $107(34.5)$ \\
\hline \multicolumn{2}{|c|}{ Primary tumor size $(\mathrm{cm})$} \\
\hline$\leq 4.5$ & $169(54.5)$ \\
\hline$>4.5$ & $141(45.5)$ \\
\hline \multicolumn{2}{|l|}{ T category } \\
\hline 1 & $8(2.6)$ \\
\hline 2 & $52(16.8)$ \\
\hline 3 & $96(31.0)$ \\
\hline 4 & $154(49.7)$ \\
\hline \multicolumn{2}{|l|}{$\mathrm{N}$ category } \\
\hline 0 & $141(45.5)$ \\
\hline 1 & $118(38.1)$ \\
\hline 2 & $51(16.5)$ \\
\hline \multicolumn{2}{|l|}{ M category } \\
\hline 0 & $170(54.8)$ \\
\hline 1 & $140(45.2)$ \\
\hline \multicolumn{2}{|l|}{ TNM stage } \\
\hline II & $101(32.6)$ \\
\hline III & $69(22.2)$ \\
\hline IV & $140(45.2)$ \\
\hline \multicolumn{2}{|c|}{ Histological grade } \\
\hline Well/moderate & 260 (83.9) \\
\hline Poor & $50(16.1)$ \\
\hline \multicolumn{2}{|l|}{ CEA level (ng/mL) } \\
\hline$\leq 5$ & $128(41.3)$ \\
\hline$>5$ & $174(56.1)$ \\
\hline Not available & $8(2.6)$ \\
\hline \multicolumn{2}{|c|}{ CA19-9 level (U/mL) } \\
\hline$\leq 35$ & $211(68.1)$ \\
\hline$>35$ & $87(28.1)$ \\
\hline Not available & $12(3.9)$ \\
\hline
\end{tabular}

CEA, carcinoembryonic antigen before primary tumor resection; CA19-9, cancer antigen 19-9 before primary tumor resection.
2-121 months), the 3 -year overall survival (OS) rate was $66.5 \%$ in all 310 patients. Patients with high FSTL1 expression in fully scanned tumors had a significantly lower 3 -year OS rate than those with low FSTL1 expression (Figure $7 A ; 72.7 \%$ vs. $62.0 \%, \mathrm{P}=0.041$ ). However, interestingly, high FSTL1 expression in the tumor stroma could indicate a better survival than low FSTL1 expression (Figure $7 B ; 57.8 \%$ vs. $76.5 \%, \mathrm{P}=0.002$ ).

As shown in Table 3, a univariate analysis of survival revealed that high FSTL1 expression in fully scanned tumor tissues ( $\mathrm{HR}=1.454$; 95\% CI: $1.013-2.088$; $\mathrm{P}=0.042$ ), low FSTL1 expression in the tumor stroma ( $\mathrm{HR}=0.577 ; 95 \%$ CI: $0.403-0.826 ; \mathrm{P}=0.003$ ), tumors located in the colon (HR $=0.563 ; 95 \%$ CI: $0.383-0.829 ; \mathrm{P}=0.004)$, primary tumor size $\leq 4 \mathrm{~cm}$ (HR $=0.698 ; 95 \%$ CI: 0.488-0.997; $\mathrm{P}=0.048), \mathrm{III} / \mathrm{IV}$ TNM stage ( $\mathrm{HR}=2.710 ; 95 \% \mathrm{CI}: 1.746-4.208 ; \mathrm{P}<0.001)$, preoperative CEA level $>5 \mathrm{ng} / \mathrm{mL}(\mathrm{HR}=2.491 ; 95 \%$ CI: 1.678-3.697; $\mathrm{P}<0.001)$ and preoperative carbohydrate antigen 19-9 (CA19-9) level $>35 \mathrm{U} / \mathrm{mL}(\mathrm{HR}=3.207$; $95 \%$ CI: 2.244-4.583; $\mathrm{P}<0.001$ ) were associated with a low OS rate. The multivariate analysis subsequently demonstrated that low FSTL1 expression in the tumor stroma $(\leq 3.17)$ (HR $=0.571 ; 95 \%$ CI: 0.395-0.826; $\mathrm{P}=0.003)$, high TNM stage (III/IV) (HR =2.631; 95\% CI: 1.681-4.117; $\mathrm{P}<0.001$ ), high preoperative CEA level $(>5 \mathrm{ng} / \mathrm{mL})(\mathrm{HR}=1.984 ; 95 \% \mathrm{CI}$ : 1.296-3.039; $\mathrm{P}=0.002)$ and high preoperative CA19-9 level $(>35 \mathrm{U} / \mathrm{mL})(\mathrm{HR}=2.387 ; 95 \%$ CI: $1.625-3.506 ; \mathrm{P}<0.001)$ were independent prognostic factors for a low OS rate.

\section{Discussion}

In the present study, we found that FSTL1 expression in clinical samples (both clinical tissues and serum samples) was higher in CRC than in corresponding normal tissues through transcriptome array and ELISA analyses; this was then validated by RT-qPCR. We conducted an IHC analysis of FSTL1 expression in CRC patients using a TMA and found that FSTL1 was expressed in both the cancer epithelial cells and the tumor stroma. When a full-area scan analysis of each stained sample was assessed, FSTL1 was found to be more highly expressed in CRC tissues than in tumor-adjacent normal tissues. However, when the focus was stromal regions in a narrow scan, FSTL1 expression was found to be significantly higher in normal tissues than in cancerous tissues. Otherwise, patients with high FSTL1 expression in tumor tissue exhibited a significantly shorter OS than patients with low FSTL1 expression. However, a narrow scan of the tumor stroma suggested that high 

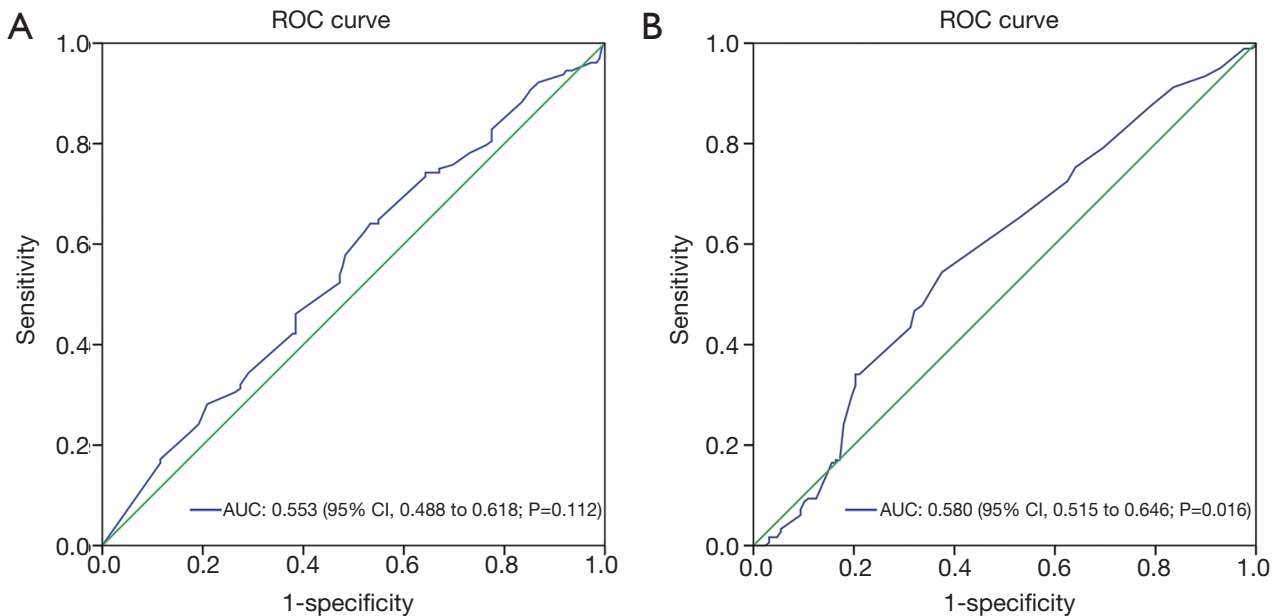

Figure 6 Optimal cutoff levels for FSTL1 expression were applied with ROC curves for overall survival. (A) ROC curve for optimal cutoff levels for FSTL1 expression in fully scanned CRC tissues according to overall survival. The optimal IHC score cutoff value was 6.5; (B) ROC curve for optimal cutoff levels for FSTL1 expression in a narrow scan of the CRC stromal region according to overall survival. The optimal IHC score cutoff value was 3.17. ROC, receiver operating characteristic; FSTL1, follistatin-like protein 1; IHC, immunohistochemistry; CRC, colorectal cancer; TMA, tissue microarray.

FSTL1 expression indicated a better overall long-term survival. Moreover, in a multivariate analysis of factors that influenced OS, we found that FSTL1 expression in the tumor stroma, but not FSTL1 in the full scan, was an independent prognostic factor, together with TNM stage, and the levels of CEA and CA19-9.

FSTL 1 is an extracellular glycoprotein and a proinflammatory molecule that promotes arthritis (19). Interestingly, the level and pattern of FSTL1 expression changes with different diseases, including cardiovascular disease $(10,20)$, systemic autoimmune diseases (21), and cancer (11). For cancer, FSTL1 has previously been reported to play both oncogenic and tumor-suppressive roles in various cancer types $(14,22)$. On the one hand, FSTL1 can drive oncogenesis and metastasis in ESCC by coordination of the NF- $\kappa \mathrm{B}$ and BMP pathways (13), and it can promote cancer cell metastasis to the bone via the inhibition of antitumor immune responses (22). Poor prognosis in glioblastoma patients due to FSTL1 overexpression was also noted (23).

On the other hand, FSTL1 was reported to suppress tumor growth via the induction of apoptosis in ovarian and endometrial cancer cells (14), and FSTL1 expression inhibited cell growth and participated in the suppression of invasiveness and metastasis of lung cancer cells (24). In our study, FSTL1 expression was validated in clinical tissues as well as serum samples. The mRNA and protein expression levels of FSTL1 were higher in tumors than in their normal counterparts. However, additional studies are needed to determine the functions of FSTL1 in CRC cells.

As a secretory protein, FSTL1 can be detected in peripheral blood serum. Serum FSTL1 levels are notably increased in patients with rheumatoid arthritis and osteoarthritis. A few studies have explored the serum level of FSTL1 protein in human cancers $(21,25)$. In esophageal squamous cell carcinoma (ESCC), FSTL1 was significantly higher in sera collected from 104 ESCC patients than in sera collected from 30 healthy individuals (22). However, Chen et al. found no significant difference in the plasma FSTL1 level between patients with colon cancer and healthy individuals (16). In our study, the FSTL1 expression level was detected in the serum of CRC patients and healthy donors. We found that FSTL1 expression was significantly higher in CRC patients than in healthy individuals. However, only 34 healthy individuals and 60 CRC patients were assessed, and thus, more participants are required to validate the results of this study. Also, the association between the FSTL1 expression level in peripheral blood serum and the clinicopathological features and prognosis needs further exploration.

Most cell lines derived from human tumors express lower levels of FSTL1 compared with immortalized nontumorigenic fibroblasts (26). Recently, Terres et al. identified FSTL1 as an upregulated stromal protein in 
Table 2 Relationships between FSTL1 expression in the tumor stroma and patient characteristics

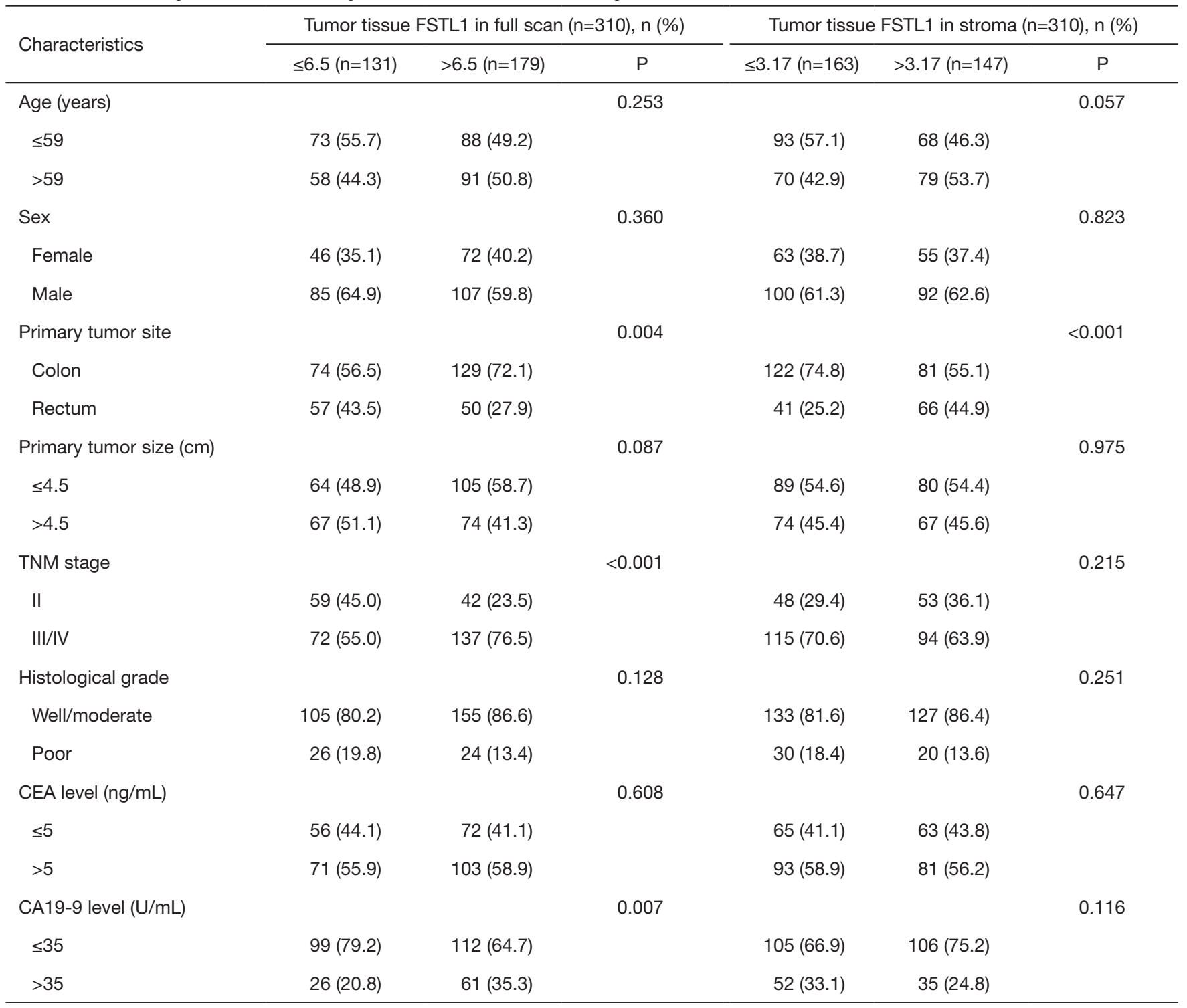

FSTL1, follistatin-like protein 1; CEA, carcinoembryonic antigen before primary tumor resection; CA19-9, cancer antigen 19-9 before primary tumor resection.

cancer-associated fibroblasts (CAFs) via an analysis of the expression profiles of normal fibroblasts (NFs) and CAFs from a mouse model of sporadic colon cancer. In contrast, they did not observe significant expression in cancer epithelial cells, and the survival analysis indicated that patients with FSTL1-positive stroma had a slightly better prognosis (with a low significance $\mathrm{P}=0.059$ ) (11). Due to the heterogeneity of different cell lines and cancers, and the complexity of the many mechanisms that underlie tumor development, our knowledge of FSTL1 in cancer development is still fragmented and limited (27). In previous studies, Gu et al. found that FSTL1 expression was significantly up-regulated in CRC tissue compared with the paired normal tissue using IHC staining in 130 paraffin-embedded CRC tissue sections (28). In our study, FSTL1 was found to be more highly expressed in tumor tissue than in their nontumor counterparts using IHC, which is consistent with the above research that FSTL1 may play a tumor-promoting role in CRC. However, based on previous studies, we also analyzed FSTL1 expression in 

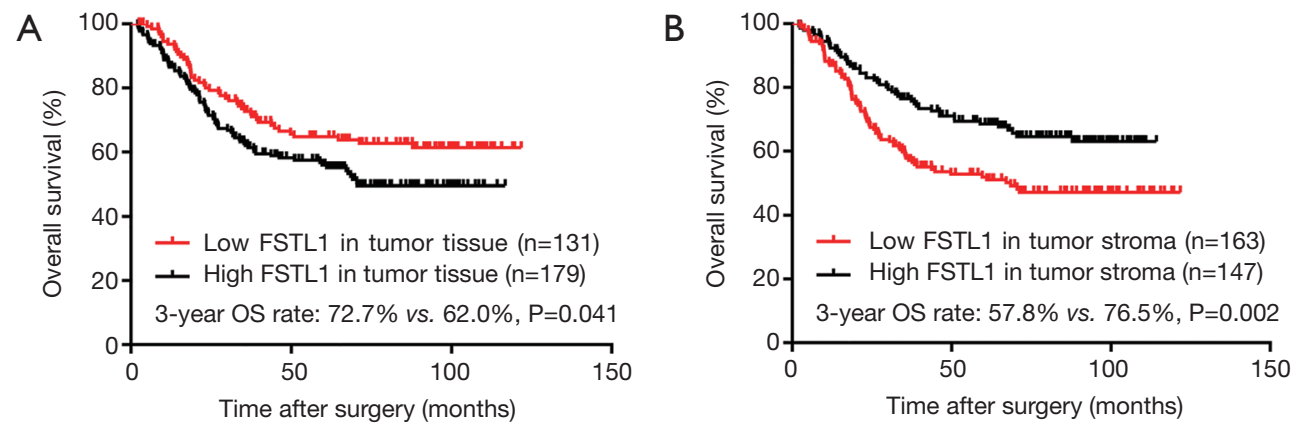

Figure 7 Kaplan-Meier survival analysis comparing the survival rate of patients with different FSTL1 expression levels. (A) Survival analysis of patients with high FSTL1 expression and low FSTL1 expression in fully scanned CRC tissues (3-year OS rate: 72.7\% vs. 62.0\%, $\mathrm{P}=0.041$ ); (B) survival analysis of patients with high FSTL1 expression and low FSTL1 expression in a narrow scan of the CRC stromal region (3-year OS rate: $57.8 \%$ vs. 76.5\%, P=0.002). FSTL1, follistatin-like protein 1; CRC, colorectal cancer; OS, overall survival.

the stroma of tumor tissues and corresponding nontumor tissues. We found the opposite result, which suggested that FSTL1 expression in the stroma was significantly higher in nontumor tissues than in the tumor tissue. How is this seemingly contradictory result explained? In previous studies, similar results were obtained in CRC patients (16). Chen et al. reported that a full image scan of CRC tissues and tumor-adjacent normal tissues showed that FSTL1 levels were higher in cancer tissues than in normal tissues, whereas a narrow scan focusing on the stromal region indicated that FSTL1 was slightly higher in normal tissues than in cancerous tissues. They also indicated that silencing of FSTL1 in the colonic fibroblast cell line CCD$18 \mathrm{Co}$ induced accelerated proliferation of colon cancer cell lines in cocultures. Exogenous FSTL1 attenuates colon cancer cell proliferation negatively. Those previous results indicate that FSTL1 exhibits a tumor suppressor effect in CRC. Why is FSTL1 higher in cancer tissues when it shows an inhibitory effect on tumor cells? A possible explanation was given in a previous study, which suggested that FSTL1 can be induced in vascular smooth muscle cells (VSMCs) and that it inhibits the proliferation of VSMCs in vitro and in vivo (9). In CRC, a possible explanation is that because FSTL1 can be induced in highly proliferative regions, it can exert antiproliferative effects. Another explanation is based on the previous study that FSTL1 may be overexpressed at tumor invasion front or stromal tissue and interacts with vimentin from the tumor tissue (28). In our study, a multivariate analysis revealed that FSTL1 expression in the tumor stroma might be a more powerful prognostic predictor in CRC patients than its expression in a full-tumor scan. All these results suggest that FSTL1 expression in the tumor stroma might be a promising prognostic biomarker for patients with CRC. Thus, further investigation is needed to confirm the role of extracellular FSTL1 and to reveal its biological functions.

Some potential limitations of the current study should be considered, however. First, the detection of FSTL1 protein in serum samples by ELISA was performed using fresh peripheral serum within two months after it was collected, which led to insufficient follow-up time. Therefore, the role of the serum level of FSTL1 protein as a prognostic predictor was not evaluated in our study. More participants and longer follow-up durations are needed in future studies. Second, although the mRNA and protein levels, as well as the locations of FSTL1, were elucidated in our study and some interesting findings were discovered, these data seemed to be insufficient to reveal the exact molecular mechanisms by which FSTL1 participates in the tumorigenesis and metastasis of CRC.

\section{Conclusions}

In conclusion, our data showed that FSTL1 is highly expressed in colorectal tumor tissues compared with tumor-adjacent normal tissue, but a narrow scan focusing on the stromal region indicated that FSTL1 expression is significantly higher in nontumor counterparts than in cancerous tissues. Furthermore, high expression in a full scan of tumor tissue and low expression in the tumor stroma both indicate a worse long-term survival. The data obtained in our study indicate that FSTL1 has obvious potential for development into a diagnostic and prognostic biomarker for patients with CRC. Therefore, the mechanism by which 
Table 3 Univariate and multivariate analyses using Cox proportional hazards models for clinicopathological factors that influence OS

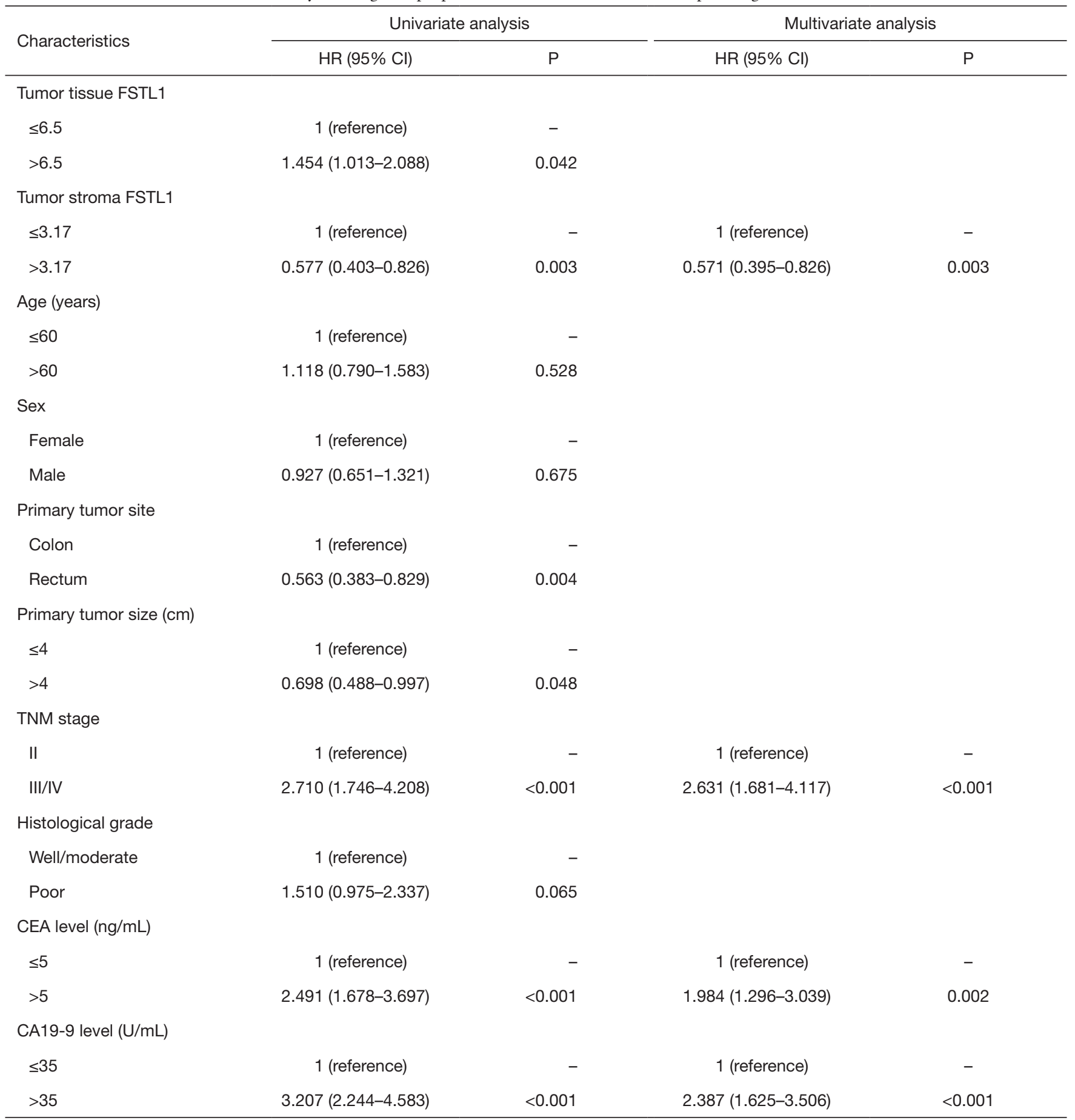

$P$ value: in the Cox hazards regression analyses, variables found to be statistically significant $(P<0.05)$ in the univariate analysis were entered into a Cox regression multivariate model using a forward conditional method. FSTL1, Follistatin-like protein 1; HR, hazard ratio; Cl, confidence interval; CEA, carcinoembryonic antigen before primary tumor resection; CA19-9, cancer antigen 19-9 before primary tumor resection; OS, overall survival. 
FSTL1 participates in CRC development warrants further exploration.

\section{Acknowledgments}

We gratefully acknowledge the assistance of colleagues in the Department of Colorectal Surgery at Sun Yat-sen University Cancer Center, who were involved in performing the treatments for the current study. The authors would like to thank the two pathologists, $\mathrm{Na}$ Guo, and Caixia Gan, from the Department of Pathology at Sun Yat-sen University Cancer Center for the IHC score evaluation.

Funding: This work was supported by grants from the National Natural Science Foundation of China (No. 81871991 and No. 81772595), the Sun Yat-sen University Clinical Research 5010 Program (No. 2015024), the Guangzhou Science and Technology Planning Projects (No. 201704030101) and the Guangzhou Science and Technology Planning Projects (Health Medical Collaborative Innovation Program of Guangzhou) (No. 201803040019).

\section{Footnote}

Conflicts of Interest: The authors have no conflicts of interest to declare.

Ethical Statement: The authors are accountable for all aspects of the work in ensuring that questions related to the accuracy or integrity of any part of the work are appropriately investigated and resolved. The present study was undertaken according to the ethical standards of the World Medical Association Declaration of Helsinki. Informed consent was waived because of the nature of retrospective study, and the patient data were kept confidentially.

\section{References}

1. Chen $\mathrm{W}$, Zheng R, Baade PD, et al. Cancer statistics in China, 2015. CA Cancer J Clin 2016;66:115-32.

2. Quah HM, Chou JF, Gonen M, et al. Identification of patients with high-risk stage II colon cancer for adjuvant therapy. Dis Colon Rectum 2008;51:503-7.

3. Lo DS, Pollett A, Siu LL, et al. Prognostic significance of mesenteric tumor nodules in patients with stage III colorectal cancer. Cancer 2008;112:50-4.

4. Chaly Y, Hostager B, Smith S, et al. Follistatin-like protein
1 and its role in inflammation and inflammatory diseases. Immunol Res 2014;59:266-72.

5. Wilson DC, Marinov AD, Blair HC, et al. Follistatin-like protein 1 is a mesenchyme-derived inflammatory protein and may represent a biomarker for systemic-onset juvenile rheumatoid arthritis. Arthritis Rheum 2010;62:2510-6.

6. Tanaka M, Ozaki S, Kawabata D, et al. Potential preventive effects of follistatin-related protein/TSC36 on joint destruction and antagonistic modulation of its autoantibodies in rheumatoid arthritis. Int Immunol 2003;15:71-7.

7. Kawabata D, Tanaka M, Fujii T, et al. Ameliorative effects of follistatin-related protein/TSC-36/FSTL1 on joint inflammation in a mouse model of arthritis. Arthritis Rheum 2004;50:660-8.

8. Le Luduec JB, Condamine T, Louvet C, et al. An immunomodulatory role for follistatin-like 1 in heart allograft transplantation. Am J Transplant 2008;8:2297-306.

9. Liu S, Wang L, Wang W, et al. TSC-36/FRP inhibits vascular smooth muscle cell proliferation and migration. Exp Mol Pathol 2006;80:132-40.

10. Oshima Y, Ouchi N, Sato K, et al. Follistatin-like 1 is an Akt-regulated cardioprotective factor that is secreted by the heart. Circulation 2008;117:3099-108.

11. Tan $X$, Zhai $Y$, Chang $W$, et al. Global analysis of metastasis-associated gene expression in primary cultures from clinical specimens of clear-cell renal-cell carcinoma. Int J Cancer 2008;123:1080-8.

12. Su S, Parris AB, Grossman G, et al. Up-Regulation of Follistatin-Like 1 By the Androgen Receptor and Melanoma Antigen-A11 in Prostate Cancer. Prostate 2017;77:505-16.

13. Kudo-Saito C, Fuwa T, Murakami K, et al. Targeting FSTL1 prevents tumor bone metastasis and consequent immune dysfunction. Cancer Res 2013;73:6185-93.

14. Chan QK, Ngan HY, Ip PP, et al. Tumor suppressor effect of follistatin-like 1 in ovarian and endometrial carcinogenesis: a differential expression and functional analysis. Carcinogenesis 2009;30:114-21.

15. Bae K, Park KE, Han J, et al. Mitotic cell death caused by follistatin-like 1 inhibition is associated with up-regulated Bim by inactivated Erk1/2 in human lung cancer cells. Oncotarget 2016;7:18076-84.

16. Chen SX, Xu XE, Wang XQ, et al. Identification of colonic fibroblast secretomes reveals secretory factors regulating colon cancer cell proliferation. J Proteomics 2014;110:155-71. 
17. Torres S, Bartolome RA, Mendes M, et al. Proteome profiling of cancer-associated fibroblasts identifies novel proinflammatory signatures and prognostic markers for colorectal cancer. Clin Cancer Res 2013;19:6006-19.

18. Tanaka M, Ozaki S, Osakada F, et al. Cloning of follistatinrelated protein as a novel autoantigen in systemic rheumatic diseases. Int Immunol 1998;10:1305-14.

19. Clutter SD, Wilson DC, Marinov AD, et al. Follistatinlike protein 1 promotes arthritis by up-regulating IFNgamma. J Immunol 2009;182:234-9.

20. Wei K, Serpooshan V, Hurtado C, et al. Epicardial FSTL1 reconstitution regenerates the adult mammalian heart. Nature 2015;525:479-85.

21. Li D, Wang Y, Xu N, et al. Follistatin-like protein 1 is elevated in systemic autoimmune diseases and correlated with disease activity in patients with rheumatoid arthritis. Arthritis Res Ther 2011;13:R17.

22. Lau MC, Ng KY, Wong TL, et al. FSTL1 Promotes Metastasis and Chemoresistance in Esophageal Squamous Cell Carcinoma through NFкB-BMP Signaling Crosstalk. Cancer Res 2017;77:5886-99.

23. Reddy SP, Britto R, Vinnakota K, et al. Novel glioblastoma markers with diagnostic and prognostic value identified through transcriptome analysis. Clin Cancer Res 2008;14:2978-87.

24. Sumitomo K, Kurisaki A, Yamakawa N, et al. Expression of a TGF-beta1 inducible gene, TSC-36, causes growth inhibition in human lung cancer cell lines. Cancer Lett 2000;155:37-46.

25. Wang Y, Li D, Xu N, et al. Follistatin-like protein 1: a serum biochemical marker reflecting the severity of joint damage in patients with osteoarthritis. Arthritis Res Ther 2011;13:R193.

26. Mattiotti A, Prakash S, Barnett P, et al. Follistatin-like 1 in development and human diseases. Cell Mol Life Sci 2018;75:2339-54.

27. Mashimo J, Maniwa R, Sugino H, et al. Decrease in the expression of a novel TGF beta1-inducible and rasrecision gene, TSC-36, in human cancer cells. Cancer Lett 1997;113:213-9.

28. Gu C, Wang X, Long T, et al. FSTL1 interacts with VIM and promotes colorectal cancer metastasis via activating the focal adhesion signalling pathway. Cell Death Dis 2018;9:654.
Cite this article as: Zhao Y, Ou Q, Deng Y, Peng J, Li C, Li J, Zhao Q, Qiu M, Wan D, Fang Y, Pan Z. Determination of follistatin-like protein 1 expression in colorectal cancer and its association with clinical outcomes. Ann Transl Med 2019;7(21):606. doi: 10.21037/atm.2019.09.20 